\title{
Recipient Vessel Thrombectomy and Anastomosis within the Zone of Injury in Microvascular Extremity Reconstruction
}

\author{
Jeremy M. Powers, MD ${ }^{1}$ Jaime H. Choi, BA ${ }^{2}$ Santosh S. Kale, MD, MBA ${ }^{1}$ \\ ${ }^{1}$ Division of Plastic and Reconstructive Surgery, Department of \\ Address for correspondence Jeremy M. Powers, MD, Division of \\ Surgery, Virginia Commonwealth University Medical Center, \\ Plastic and Reconstructive Surgery, VCU, PO Box 980154, 1250 E \\ Richmond, Virginia \\ 2 Virginia Commonwealth University School of Medicine, Richmond,
} Virginia

J Reconstr Microsurg Open 2020;5:e15-e21.

\begin{abstract}
Keywords

- lower extremity reconstruction

- microvascular reconstruction

- zone of injury

- thrombectomy

- vessel preparation

Background In traumatic extremity reconstruction, it is preferable to perform microvascular anastomoses outside the zone of injury. Alternatively, a recipient-vessel thrombectomy can be performed to improve the arterial inflow, potentially avoiding further proximal dissection and the use of a vein graft. The objective of this study was to evaluate the outcomes of microvascular anastomosis after thrombectomy in the zone of injury.

Methods A retrospective review of patients who underwent free tissue transfer for traumatic extremity reconstruction by the senior author from 2013 to 2017 was conducted. Patient demographic and clinical data were gathered as well as use of vein grafts, recipient-vessel thrombectomy, postoperative anticoagulation, and flap outcomes.

Results A total of 23 patients underwent 24 free flap procedures for traumatic extremity reconstruction. Ten patients underwent recipient-vessel thrombectomy with 2-Fr Fogarty catheter and were placed on postoperative anticoagulation. In this group overall, there was one case of flap loss due to late wound infection resulting in a $90 \%$ success rate. A total of 13 patients underwent 14 free flaps without the use of thrombectomy and vein grafts were performed for $3 / 14$ (21\%) patients. In this group, there were four cases of flap loss, resulting in a $71 \%$ overall success rate.

Conclusion In traumatic extremity reconstruction, if the artery appears to be visibly appropriate with weak inflow, one may consider attempting thrombectomy to restore pulsatile flow prior to harvesting a vein graft. This study suggests this may be effective and safe with no anastomosis-related flap loss in the thrombectomy group. Further research is required to confirm our results.
\end{abstract}

Managing severe extremity trauma involving extensive soft tissue and bony injuries is challenging for even the experienced reconstructive surgeon. With overall success rates exceeding $90 \%$, microvascular free tissue transfer has be- come the gold standard for approaching massive wounds. ${ }^{1,2}$ Compared with alternative methods, such as local/pedicled flaps, free tissue transfer has the ability to bring a large amount of nontraumatized tissue to provide for optimal received

January 6, 2020

accepted

March 20, 2020
DOI https://doi.org/

10.1055/s-0040-1710347. ISSN 2377-0813.
Copyright $\odot 2020$ by Thieme Medical Publishers, Inc., 333 Seventh Avenue, New York, NY 10001, USA. Tel: +1(212) 760-0888.
License terms

()(1) $\Theta \circledast$ 
wound healing and decreased risk of infection. ${ }^{1}$ Typically, extremity wounds resulting in the need for free tissue transfer are high-energy complex injuries involving an entire circumferential segment of the limb and all structures that traverse the area. ${ }^{3,4}$

The injured segment creates a "zone of injury" that has been defined as "the inflammatory response of the soft tissue of the traumatized (region) that extends beyond the gross wound and results in perivascular changes in the blood vessels ${ }^{4}$." It has long been held that it is preferable to perform microvascular anastomoses with vessels that are outside of the zone of injury, as endothelial damage as well as the inflammatory milieu in this zone is thought to promote thrombosis. ${ }^{5-7}$ Indeed, failure to recognize the true extent of injury has been implicated as one of the main contributors to thrombosis and flap failure after microvascular anastomosis. ${ }^{5-7}$

These concepts form the basis for recommendations by leading experts that encourage surgeons to utilize vessels outside the zone of injury to achieve microvascular anastomosis with minimal complications. ${ }^{3,5,8,9}$ This may require extensive proximal dissection, alternative retrograde inflow distal to the zone of injury, ${ }^{2,3}$ harvest of increased pedicle length, and the possible use of interpositional vein grafts. ${ }^{10}$ However, more complex cases, such as those involving massive trauma, limited donor sites, certain anatomic constraints, and/or limited pedicle lengths, may make pursuit of anastomosis outside the zone of injury unsuitably morbid. ${ }^{2}$

Regardless of location, a vessel must have adequate flow to complete a successful microanastomosis. This is usually assessed intraoperatively with visual inspection for pulsatile flow. In situations of inadequate pulsatile flow, current practice algorithms describe further proximal or distal dissection to uninjured vessel with or without the use of an interpositional vein graft. ${ }^{1,11-13}$ The primary purpose of this study was to assess the outcomes of free flap reconstructions among patients who underwent a vessel salvage protocol consisting of balloon angioplasty with thrombectomy until restoration of pulsatile flow, timely completion of microanastomosis within the zone of injury, and placement on pharmacologic anticoagulation using a heparin drip. These patients were compared with a group who underwent proximal anastomosis with or without vein grafts and postoperative anticoagulation.

\section{Methods}

\section{Study Design and Data Analysis}

Following approval by the Virginia Commonwealth University Institutional Review Board, a retrospective chart review was performed for all patients of the senior author undergoing free tissue transfer for traumatic lower or upper extremity reconstruction from 2013 to 2017. The sample was derived from a single surgeon's practice at Virginia Commonwealth University Medical Center, an academic Level I trauma center. The majority of patients presented with Gustilo-Anderson Grade IIIB defects of the lower extremity. Preoperative history and physical, operative reports, and postoperative follow-up notes were reviewed for data collec- tion. Variables collected included basic patient demographic characteristics, medical comorbidities, wound location, fracture type, presence of osteomyelitis, flap type, time until reconstruction, time in practice, ischemia time, return to operating room, early and late postoperative complications, and whether or not recipient-vessel arterial thrombectomy, vein grafts, or postoperative anticoagulation were used. Data were collected in an Excel spreadsheet (Microsoft, Redmond, WA) and both descriptive and analytic statistics were performed with SPSS (IBM, Armonk, New York). Pearson's Chisquare and Fischer's exact tests were used for categorical variables and two-tailed $t$-tests were used for comparison of means of continuous variables. A $p$-value of $<0.05$ was considered statistically significant.

\section{Surgical Algorithm}

Our surgical technique began with thorough debridement of the open wound, followed by exposure of the anterior or posterior tibial arteries within or subjacent to the zone of injury. Grossly uninjured vessels as seen under $3.5 \times$ loupe magnification with nonfriable adventitia, no ecchymosis and no evidence of thrombosis were transected and assessed for pulsatile flow. With presence of robust pulsatile flow, microvascular anastomosis would proceed in end-to-end fashion with standard suture technique ( $\mathbf{- F i g . 1}$ ). With sluggish or nonpulsatile flow, heparinized saline and topical antispasmodic agents are used and if effective in restoring blood flow, anastomosis would proceed.

If the vessel remained nonpulsatile after the use of antispasmodic agents, we performed balloon angioplasty and arterial thrombectomy with a 2-French Fogarty arterial embolectomy balloon catheter (Edward's Lifesciences, Irvine, CA) in an attempt to reestablish pulsatile flow. If successful in restoring blood flow, microvascular anastomosis would proceed in an end-to-end fashion using standard technique. After the additional vessel manipulation, pharmacologic anticoagulation with a heparin drip would be started, with an initial rate of 18 units $/ \mathrm{kg} / \mathrm{h}$ and a goal partial thromboplastin time of 70 to 110 . If unsuccessful after up to two attempts, we proceeded with the standard technique of proximal dissection, resection of injured vessel segment, and if needed, utilization of an interpositional vein graft. ${ }^{10}$

\section{Results}

A total of 23 patients were identified in this study, with 10 patients who received thrombectomy and 13 patient who did not receive a thrombectomy. One patient who did not receive thrombectomy underwent two free flap procedures, yielding 14 total free flap reconstructions in this group. All patients who did receive thrombectomy underwent one free flap procedure, yielding 10 total free flaps in this group. Patient demographic data are summarized in - Table 1. Medical comorbidities including diabetes, peripheral vascular disease, presence of osteomyelitis, and smoking did not significantly differ between the two groups (-Table $\mathbf{1}$ ).

Surgical details are found in - Table 2. While all patients who underwent thrombectomy solely had lower extremity 


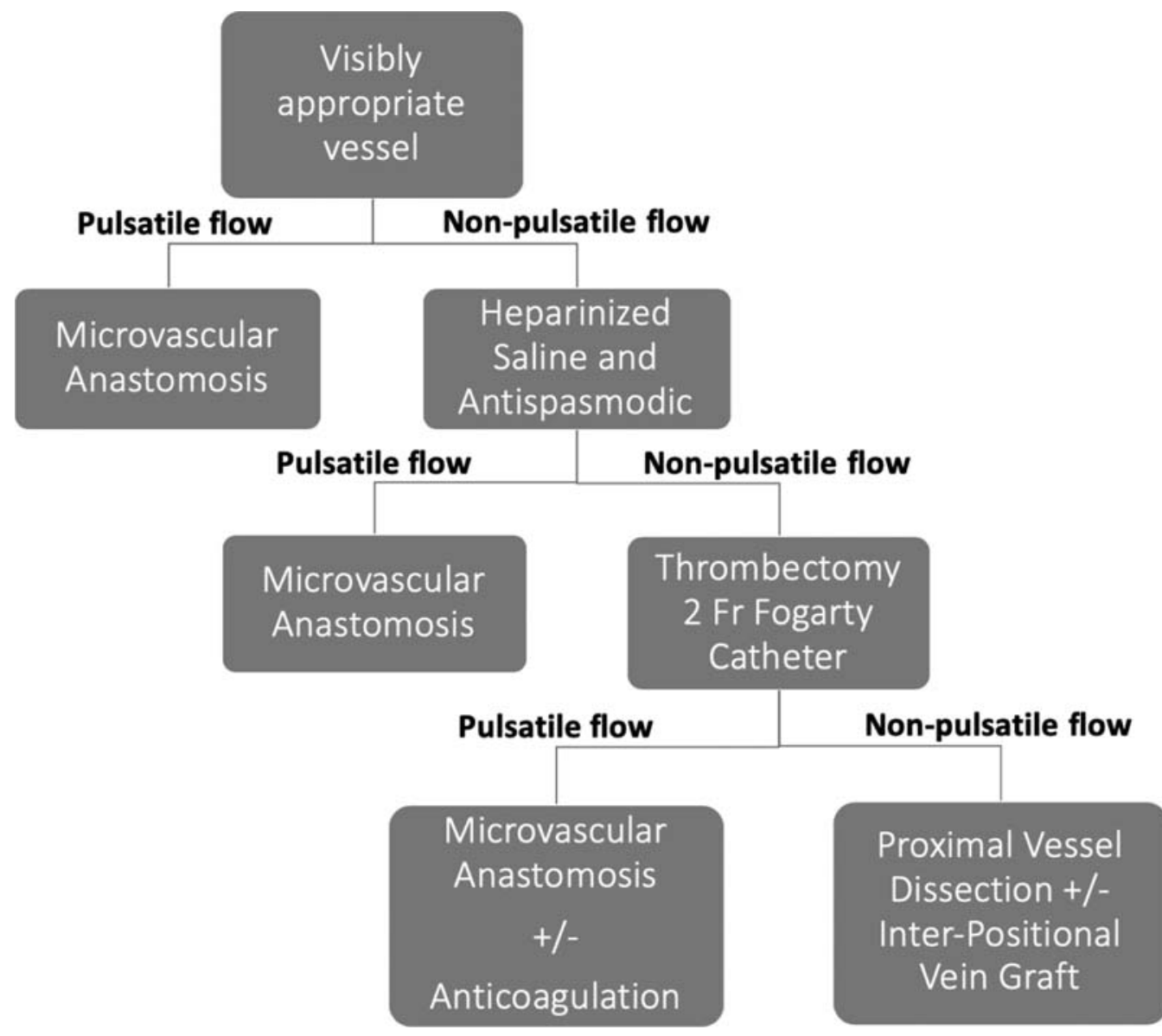

Fig. 1 Surgical algorithm for recipient vessel preparation.

reconstructions, patients who did not receive thrombectomy underwent a mix of upper $(5 / 13,38 \%)$ and lower $(9 / 13,62 \%)$ extremity reconstructions $(p<0.05)$. The median time to reconstruction was not significantly different in patients without thrombectomy compared with patient with thrombectomy ( 15 vs. 26 days; $p=0.408$ ). The mean ischemia time in patients without thrombectomy was 88 minutes compared with 124 minutes in those with thrombectomy $(p=0.065)$.

Choice of flap was similar between the two groups, with the majority of patients receiving anterolateral thigh (ALT) flaps (64\% in the no thrombectomy group and $80 \%$ in the thrombectomy group). The deep inferior epigastric artery perforator flap was used in $3 / 14$ (21\%) reconstructions in the thrombectomy group, and in one patient in whom thrombectomy was utilized. The tensor fascia lata (TFL) perforator flap was used in one patient in the thrombectomy group, and no patients in the no thrombectomy group. Recipient artery selection included anterior or posterior tibial arteries for lower extremity reconstructions, depending on the location of the defect, as well as radial or ulnar artery for the upper extremity reconstructions. Recipient vein selection included the vena comitans of the selected artery, along with an additional anastomosis to the superficial system in 5/14 (36\%) reconstructions in the no thrombectomy group and in $5 / 10(50 \%)$ reconstructions in the thrombectomy group.

Table 1 Patient demographics

\begin{tabular}{|l|l|l|}
\hline & No thrombectomy $(\boldsymbol{n}=\mathbf{1 4})$ & Thrombectomy $(\boldsymbol{n}=\mathbf{1 0})$ \\
\hline Age in years mean (standard deviation) & $40(15.6)$ & $42(16.1)$ \\
\hline Male & $71 \%$ & $90 \%$ \\
\hline Current smokers & $36 \%$ & $50 \%$ \\
\hline Diabetes & $14 \%$ & \\
\hline Hypertension & $14 \%$ & $10 \%$ \\
\hline Peripheral vascular disease & $7 \%$ & $10 \%$ \\
\hline Chronic kidney disease & 0 & \\
\hline Osteomyelitis & $7 \%$ & \\
\hline
\end{tabular}


Table 2 Surgical details

\begin{tabular}{|c|c|c|}
\hline & No thrombectomy $(n=14)$ & Thrombectomy $(n=10)$ \\
\hline Median time to reconstruction (d) & 15 & 26 \\
\hline Upper extremity $^{\mathrm{a}}$ & $36 \%$ & - \\
\hline Lower extremity $^{\mathrm{a}}$ & $64 \%$ & $100 \%$ \\
\hline \multicolumn{3}{|l|}{ Flap } \\
\hline ALT & $71 \%$ & $80 \%$ \\
\hline DIEP & $29 \%$ & $10 \%$ \\
\hline TFL & - & $10 \%$ \\
\hline \multicolumn{3}{|l|}{ Recipient artery } \\
\hline Anterior tibial & $14 \%$ & $60 \%$ \\
\hline Posterior tibial & $36 \%$ & $40 \%$ \\
\hline Radial & $33 \%$ & - \\
\hline Ulnar & $7 \%$ & - \\
\hline Other & $14 \%$ & - \\
\hline Time in practice $(d)^{a}$ & 410 & 854 \\
\hline Mean ischemia time (mi) & 88 & 124 \\
\hline Postoperative anticoagulation & $79 \%$ & $100 \%$ \\
\hline \multicolumn{3}{|l|}{ Reexploration } \\
\hline Early (<24 h) & $21 \%$ & $0 \%$ \\
\hline Late $(>24 \mathrm{~h})$ & $36 \%$ & $20 \%$ \\
\hline \multicolumn{3}{|l|}{ Flap outcomes } \\
\hline Partial loss & $7 \%$ & $0 \%$ \\
\hline Complete loss & $29 \%$ & $10 \%$ \\
\hline Overall success rate & $71 \%$ & $90 \%$ \\
\hline
\end{tabular}

Abbreviations: ALT, anterolateral thigh; DIEP, deep inferior epigastric perforator; TFL, tensor fascia lata. ${ }^{\mathrm{a}} \mathrm{p}<0.05$.

Vein grafts were used for the arterial anastomosis in $3 / 14$ (21\%) reconstructions in the no thrombectomy group. One patient in the thrombectomy group underwent balloon angioplasty and thrombectomy to the posterior tibial artery, without adequate restoration of inflow. A saphenous vein graft to the superficial femoral artery was performed with successful flap outcome despite early reoperation for hematoma. Thus, thrombectomy was effective in restoring inflow in 9/10 (90\%) recipient vessels.

Postoperative anticoagulation in the form of a heparin drip was employed in 6/14 flaps (43\%) that did not receive a thrombectomy at the surgeon's discretion, compared with all flaps in patients who did receive thrombectomy (10/10, $100 \%$ ), given the additional vessel manipulation. In patients in whom thrombectomy was not utilized, 3/14 (21\%) flaps required reexploration within the first 24 hours and $4 / 14$ (29\%) underwent reexploration after 24 hours. No thrombectomy patients required early ( $<24$ hours) reexploration, while $2 / 10(20 \%)$ patients required late reexploration for bleeding and infection.

Overall flap success rate was higher in patients who underwent thrombectomy compared with those who did not; however, this difference did not reach statistical signifi- cance ( 90 vs. $71 \% ; p=0.358$ ). The four patients in the no thrombectomy group-who sustained complete flap losswere found to have arterial thrombosis, combined arterial and venous thrombosis, isolated venous thrombosis, and hematoma resulting in flap compromise, respectively. The single incident of complete flap loss in the thrombectomy group was due to a late wound infection. There was one episode of partial flap loss in the no thrombectomy group due to a wound infection with Staphylococcus aureus; however, the necessary debridement resulted in exposed bone and required a second free flap which was successful. The use of angioplasty/thrombectomy for recipient vessel preparation represents an evolution in the senior author's practice, as reflected in the significant difference in the time in practice when comparing the two groups (average of 410 days for no thrombectomy group vs. 854 days in the thrombectomy group; $p=0.001$ ).

\section{Discussion}

The primary purpose of this study was to assess the outcomes of free flap reconstructions among patients who underwent a vessel salvage protocol consisting of balloon 
angioplasty with thrombectomy until restoration of pulsatile flow, timely completion of microanastomosis within the zone of injury, and placement on pharmacologic anticoagulation using a heparin drip. Vessel manipulation proceeded in a stepwise fashion beginning with topical antispasmodic agents and proceeding to angioplasty/thrombectomy. If neither of these were successful in restoring pulsatile flow, further proximal dissection was performed and flap anastomosis was completed with or without the use of a vein graft. Patients who received a recipient vessel thrombectomy were compared with those who did not. These data from our limited retrospective review may suggest that the technique and proposed protocol may be safe and effective with $90 \%$ overall flap survival, but further research must be conducted to validate our results.

Utilizing recipient vessels outside the zone of injury remains the preferred choice in traumatic extremity reconstruction. Determining the boundaries of the "zone of injury," however, can be difficult in situations of extensive trauma, acute or chronic inflammation/infection, and/or preexisting peripheral vascular disease. Indeed, there is little scientific data available to formulate clear criteria defining this region. ${ }^{4,8}$ Using the classic metaphor of the iceberg, Spector describes the zone of injury in lower extremity trauma as a "thrombogenic region that often spans beyond what is apparent on the surface of the wound ${ }^{3}$." Thus, accurately identifying the zone of injury is a critical step in the process of flap reconstruction of extremity wounds to avoid complications and optimize outcome. , $7,8,14^{-14}$

However, several studies have suggested that anastomosis within the zone of injury (without additional maneuvers to improve blood flow) or distal to the zone of injury (depending on reverse blood flow) can be performed without an increase in complication rate. ${ }^{2,3,8,15,16}$

An early study by Stompro and Stevenson demonstrated the feasibility of performing anastomosis distal to the zone of injury. ${ }^{15}$ They documented a success rate of $91 \%$ in a case series of 23 distally based free flap reconstructions. Another group at New York University (NYU) has also published multiple times about the use of anastomosis distal to the zone of injury for lower extremity reconstruction. ${ }^{2,3}$ The more recent review in 2007 by Spector and others compares the recent experience from 1995 to 2005 to the previously published data from Kolker from 1979 to 1995. From 1995 to 2005,28 out of 119 (24\%) of free flap reconstructions were performed with vessels distal to the zone of injury. One flap failed, representing an overall success rate of $96 \%$, which was not significantly different from the group who underwent proximal anastomosis (87 out of 119 flaps, success rate of $91 \%$ ). Combined with the prior data from 1979 to 1995 , the success rate for the flaps with distal anastomoses increased to $97 \%$. A further update of the NYU database in 2019 as well as a systematic review of the literature corroborated these results, with no difference in return to the operating room, partial flap failure, or total flap failure between proximal and distal anastomoses. ${ }^{17}$ The authors advocate routine use of magnetic resonance, computed tomography, or conventional angiography to assess for flow traversing the zone of injury and in appropriate patients, distal vessels may be selected for anastomosis with similar success rates to proximal vessel anastomosis. $^{3,17}$

Isenberg and Sherman (1996) prospectively studied 28 patients in whom lower extremity free flap reconstructions were performed. ${ }^{8}$ They measured the distance from the anastomosis to the proximal extent of the bony injury (average distance of $4.6 \mathrm{~cm}$ ) and determined that they were likely within or at least "subjacent" to the macroscopic zone of injury. Despite the proximity, the vessels utilized were documented to have minimal to no friability, perivascular scar tissue, or vessel wall thickening, and there were no increased complications in this series. The authors urged that analysis of recipient vessel characteristics, rather than actual location in relation to the wound, was more clinically relevant. $^{8}$

A more recent study corroborating these results investigated the use of vessels within the macroscopic zone of injury for free flap anastomosis. ${ }^{16}$ The authors undertook a retrospective review and compared patients who underwent anastomosis proximal to, distal to, or within the zone of injury. Recipient vessels were selected based on those in closest proximity to the defect, allowing for correct orientation/positioning for the free flap pedicle. Vessels were used even if the surrounding adventitia appeared injured or hemorrhagic. If the flow was inadequate, they state that "proximal vessel injury was studied and corrected when required" but do not mention what techniques were used. Among 48 total free flaps, 21 flaps were anastomosed proximal to the zone of injury, 5 flaps were anastomosed distal to the zone of injury, and 22 flaps were anastomosed within the zone of injury. There were no failures in the proximal group, one in the distal group and two in the within the zone of injury group, with no significant difference in the success rate between the three groups. The authors suggest that the advantages of proximal anastomosis may not outweigh the additional dissection, tunneling, and possible need for vein grafts required if that technique is utilized. They advocate a customized approach tailored to the defect, surgeon experience, and flap choice. Examples included suggesting end-to-side anastomosis within the zone of injury for a gracilis flap with its more centrally located pedicle, often oriented perpendicular to axial vessels, versus end-toend anastomosis proximal or distal to the zone of injury for a latissimus flap with its more proximal pedicle located at the periphery of the defect. ${ }^{16}$

Taken together, there seems to be sufficient evidence from clinical studies to demonstrate the safety of anastomosis proximal to, distal to, or even within the zone of injury. Regardless of the location of anastomosis, the inflow must be adequate to support the free flap. The use of our protocol involving thrombectomy and postoperative anticoagulation may have resulted in improved flap success rates in our series. The patients who did not receive a thrombectomy represented patients managed earlier in the senior authors practice on average. The overall flap success rate in this group was $71 \%$, lower than the 80 to $>95 \%$ rates reported in the 
literature for lower extremity reconstruction..$^{4,17-21}$ In the group in which the thrombectomy protocol was employed, the flap survival increased to $90 \%$ and there were no anastomotic-related complications in that group. In a small, retrospective study of this nature, it is difficult to identify the specific factors contributing to increased flap survival in the thrombectomy group. While the increase in flap survival in a single-surgeon cohort could be attributed to advances in surgeon experience and improved decision making, the use of recipient vessel balloon angioplasty and thrombectomy does not seem to have negatively impacted flap outcomes. Indeed, the question arose whether or not the use of the technique may have improved outcomes in the earlier cohort of patients. The study was limited by small sample size, retrospective nature, and single surgeon experience. Future prospective studies are required to investigate the multiple factors of surgical decision making involved in complex extremity reconstruction, including assessment of the zone of injury, preparation of the recipient vessels, selection of the ideal flap donor site, completion of the micro-anastomosis, and postoperative management.

While not previously described in the literature for patients undergoing free flap reconstructions for traumatic extremity defects, the use of Fogarty catheters in vessel preparation is a crucial tool in the reconstructive microsurgeon's armamentarium (Scott Levin, personal communication). We believe this technique may represent a useful modality to restore sufficient flow prior to microvascular anastomosis and our protocol may enable its safe application.

\section{Conclusion}

When patients present with massive extremity trauma, the mandate to go outside the zone of injury often requires significant proximal recipient vessel dissection and/or additional donor site morbidity from obtaining greater pedicle length or harvesting interpositional vein grafts. The decision to use a proximal vessel that is clearly uninjured must be balanced with the additional morbidity and technical difficulties of performing such an anastomosis. After identification of an appropriate vessel within or outside of the zone of injury, we propose a protocol of stepwise increases in vessel manipulation, which if necessary, may include the use of a 2Fr Fogarty balloon catheter for recipient-artery angioplasty and thrombectomy, followed by postoperative anticoagulation if deemed necessary ( $\mathbf{- F i g . 1}$ ). This study suggests that this protocol may be safe with improved flap survival over a single surgeon's experience, though additional research is required to confirm our results.

Note

This study was presented at American Society for Reconstructive Microsurgery 2018 in Phoenix, AZ.

\section{Funding}

None.
Conflict of Interest

None declared.

\section{References}

1 Saint-Cyr M, Wong C, Buchel EW, Colohan S, Pederson WC. Free tissue transfers and replantation. Plast Reconstr Surg 2012;130 (06):858e-878e

2 Kolker AR, Kasabian AK, Karp NS, Gottlieb JJ. Fate of free flap microanastomosis distal to the zone of injury in lower extremity trauma. Plast Reconstr Surg 1997;99(04):1068-1073

3 Spector JA, Levine S, Levine JP. Free tissue transfer to the lower extremity distal to the zone of injury: indications and outcomes over a 25-year experience. Plast Reconstr Surg 2007;120(04): 952-959

4 Loos MS, Freeman BG, Lorenzetti A. Zone of injury: a critical review of the literature. Ann Plast Surg 2010;65(06): 573-577

5 Acland RD. Refinements in lower extremity free flap surgery. Clin Plast Surg 1990;17(04):733-744

6 Francel TJ, Vander Kolk CA, Hoopes JE, Manson PN, Yaremchuk MJ. Microvascular soft-tissue transplantation for reconstruction of acute open tibial fractures: timing of coverage and long-term functional results. Plast Reconstr Surg 1992;89(03):478-487, discussion 488-489

7 Hammert WC, Minarchek J, Trzeciak MA. Free-flap reconstruction of traumatic lower extremity wounds. Am J Orthop 2000;29(09): $22-26$

8 Isenberg JS, Sherman R. Zone of injury: a valid concept in microvascular reconstruction of the traumatized lower limb? Ann Plast Surg 1996;36(03):270-272

9 Miyamoto S, Takushima A, Okazaki M, Ohura N, Minabe T, Harii K. Relationship between microvascular arterial anastomotic type and area of free flap survival: comparison of end-to-end, end-toside, and retrograde arterial anastomosis. Plast Reconstr Surg 2008;121(06):1901-1908

10 Nelson JA, Fischer JP, Grover R, et al. Vein grafting your way out of trouble: Examining the utility and efficacy of vein grafts in microsurgery. J Plast Reconstr Aesthet Surg 2015;68(06): 830-836

11 Heller L, Levin LS. Lower extremity microsurgical reconstruction. Plast Reconstr Surg 2001;108(04):1029-1041, quiz 1042

12 Parrett BM, Matros E, Pribaz JJ, Orgill DP. Lower extremity trauma: trends in the management of soft-tissue reconstruction of open tibia-fibula fractures. Plast Reconstr Surg 2006;117(04):1315-1322, discussion 1323-1324

13 Ong YS, Levin LS. Lower limb salvage in trauma. Plast Reconstr Surg 2010;125(02):582-588

14 Roehl KR, Mahabir RC. A practical guide to free tissue transfer. Plast Reconstr Surg 2013;132(01):147e-158e

15 Stompro BE, Stevenson TR. Reconstruction of the traumatized leg: use of distally based free flaps. Plast Reconstr Surg 1994;93(05): 1021-1025, discussion 1026-1027

16 Bendon CL, Giele HP. Success of free flap anastomoses performed within the zone of trauma in acute lower limb reconstruction. J Plast Reconstr Aesthet Surg 2016;69(07):888-893

17 Stranix JT, Borab ZM, Rifkin WJ, et al. Proximal versus distal recipient vessels in lower extremity reconstruction: a retrospective series and systematic review. J Reconstr Microsurg 2018;34 (05):334-340

18 Cho EH, Shammas RL, Carney MJ, et al. Muscle versus fasciocutaneous free flaps in lower extremity traumatic reconstruction: a multicenter outcomes analysis. Plast Reconstr Surg 2018;141 (01):191-199

19 Song CT, Koh K, Tan B-K, Goh T. Free-flap lower extremity reconstruction: a cohort study and meta-analysis of flap 
anastomotic outcomes between perforator and nonperforator flaps. J Reconstr Microsurg 2018;34(06):455-464

20 Ricci JA, Abdou SA, Stranix JT, et al. Reconstruction of gustilo type IIIC injuries of the lower extremity. Plast Reconstr Surg 2019;144 (04):982-987
21 Haddock NT, Weichman KE, Reformat DD, Kligman BE, Levine JP, Saadeh PB. Lower extremity arterial injury patterns and reconstructive outcomes in patients with severe lower extremity trauma: a 26-year review. J Am Coll Surg 2010;210 (01):66-72 